\title{
LINEAR FRACTIONAL RELATIONS IN BANACH SPACES: INTERIOR POINTS IN THE DOMAIN AND ANALOGUES OF THE LIOUVILLE THEOREM
}

\author{
M. I. OSTROVSKII* \\ Department of Mathematics and Computer Science, St. John's University, 8000 Utopia Parkway, \\ Queens, NY11439, USA \\ e-mail: ostrovsm@stjohns.edu
}

(Received 9 June, 2006; revised 6 December, 2006; accepted 9 January, 2007)

\begin{abstract}
In this paper we study linear fractional relations defined in the following way. Let $\mathcal{B}_{i}, \mathcal{B}_{i}^{\prime}, i=1,2$, be Banach spaces. We denote the space of bounded linear operators by $\mathcal{L}$. Let $T \in \mathcal{L}\left(\mathcal{B}_{1} \oplus \mathcal{B}_{2}, \mathcal{B}_{1}^{\prime} \oplus \mathcal{B}_{2}^{\prime}\right)$. To each such operator there corresponds a $2 \times 2$ operator matrix of the form

$$
T=\left(\begin{array}{ll}
T_{11} & T_{12} \\
T_{21} & T_{22}
\end{array}\right),
$$

where $T_{i j} \in \mathcal{L}\left(\mathcal{B}_{j}, \mathcal{B}_{i}^{\prime}\right), i, j=1,2$. For each such $T$ we define a set-valued map $G_{T}$ from $\mathcal{L}\left(\mathcal{B}_{1}, \mathcal{B}_{2}\right)$ into the set of closed affine subspaces of $\mathcal{L}\left(\mathcal{B}_{1}^{\prime}, \mathcal{B}_{2}^{\prime}\right)$ by

$$
G_{T}(K)=\left\{K^{\prime} \in \mathcal{L}\left(\mathcal{B}_{1}^{\prime}, \mathcal{B}_{2}^{\prime}\right): T_{21}+T_{22} K=K^{\prime}\left(T_{11}+T_{12} K\right)\right\} .
$$

The map $G_{T}$ is called a linear fractional relation.

The paper is devoted to the following two problems.

- Characterization of operator matrices of the form (*) for which the set $G_{T}(K)$ is non-empty for each $K$ in some open ball of the space $\mathcal{L}\left(\mathcal{B}_{1}, \mathcal{B}_{2}\right)$.

- Characterizations of quadruples $\left(\mathcal{B}_{1}, \mathcal{B}_{2}, \mathcal{B}_{1}^{\prime}, \mathcal{B}_{2}^{\prime}\right)$ of Banach spaces such that linear fractional relations defined for such spaces satisfy the natural analogue of the Liouville theorem "a bounded entire function is constant".
\end{abstract}

2000 Mathematics Subject Classification. 47A56, 46B20, 47B50.

1. Introduction. Consider a bounded linear operator $T$ between Banach spaces $\mathcal{B}, \mathcal{B}^{\prime}$ which can be decomposed into direct sums $\mathcal{B}=\mathcal{B}_{1} \oplus \mathcal{B}_{2}, \mathcal{B}^{\prime}=\mathcal{B}_{1}^{\prime} \oplus \mathcal{B}_{2}^{\prime}$. Such a linear operator can be represented by a $2 \times 2$ operator matrix of the form

$$
T=\left(\begin{array}{ll}
T_{11} & T_{12} \\
T_{21} & T_{22}
\end{array}\right),
$$

where $T_{i j} \in \mathcal{L}\left(\mathcal{B}_{j}, \mathcal{B}_{i}^{\prime}\right),(i, j=1,2)$. (By $\mathcal{L}\left(\mathcal{B}_{j}, \mathcal{B}_{i}^{\prime}\right)$ we denote the space of bounded linear operators acting from $\mathcal{B}_{j}$ to $\mathcal{B}_{i}^{\prime}(i, j=1,2)$.) The map $G_{T}$ from $\mathcal{L}\left(\mathcal{B}_{1}, \mathcal{B}_{2}\right)$ into the set of

\footnotetext{
* Supported by St. John's University Summer 2006 Support of Research Program.

The author thanks V. A. Khatskevich for suggesting him the problems considered in this paper, V. S. Shulman for his advice and encouragement, and the referee for his helpful critical comments.
} 
closed affine subspaces of $\mathcal{L}\left(\mathcal{B}_{1}^{\prime}, \mathcal{B}_{2}^{\prime}\right)$, defined by

$$
G_{T}(X)=\left\{Y \in \mathcal{L}\left(\mathcal{B}_{1}^{\prime}, \mathcal{B}_{2}^{\prime}\right): T_{21}+T_{22} X=Y\left(T_{11}+T_{12} X\right)\right\}
$$

is called a linear fractional relation (LFR) associated with $T$. The set of all $X \in \mathcal{L}\left(\mathcal{B}_{1}, \mathcal{B}_{2}\right)$ satisfying $G_{T}(X) \neq \varnothing$ is called the domain of $G_{T}$ and is denoted by $\operatorname{dom} G_{T}$.

Such relations can be considered as a generalization of linear fractional transformations which are defined in the case when the 'denominator' $\left(T_{11}+T_{12} X\right)$ is invertible, by the formula

$$
H_{T}(X)=\left(T_{21}+T_{22} X\right)\left(T_{11}+T_{12} X\right)^{-1} .
$$

It is clear by comparing the defining formulas, that for invertible $\left(T_{11}+T_{12} X\right)$ the operator $H_{T}(X)$ is the only element of $G_{T}(X)$, and the mappings can be identified.

M. G. Krein [12] found applications of operator linear fractional transformations to the theory of spaces with an indefinite metric. The theory of linear fractional transformations was developed further in [13]. Now linear fractional transformations are used in many different contexts (see references in the introduction of [8]). In some situations the requirement of invertibility of the operator $\left(T_{11}+T_{12} X\right)$ (needed to define a linear fractional transformation) does not come from the nature of the problem and narrows the applicability of the results obtained. For this reason it is natural to develop a (more general) theory of linear fractional relations, and to prove some of the known results in more general form. Some steps in this direction have already been done. (See [7], [8], [9], and references therein.) Another direction of generalization of the classical results is to consider the Banach space case (see [1, pp. 448-449], [3], [4], [9], [10], [11], and references therein). In this paper we combine these directions.

The paper [8] contains, in the Hilbert space case, a characterization of operators $T$ for which the domain of $G_{T}$ contains interior points. The main purpose of the present paper is to generalize these results of [8] to the Banach space case. We use the Banach space theory terminology and notation from [14]. For a Banach space $X$ we denote by $B_{X}(r)$ the set $\{x \in X:\|x\| \leq r\}$; the unit ball (corresponding to $r=1$ ) is denoted by $B_{X}$. If $X$ is not specified, we mean the space $\mathcal{L}\left(\mathcal{B}_{1}, \mathcal{B}_{2}\right)$. We say that a continuous linear operator acting between two Banach spaces is left Fredholm if its kernel is finite dimensional and its image is closed.

Definition 1. A linear fractional relation $G_{T}$ is called constant if $\operatorname{dom} G_{T}=$ $\mathcal{L}\left(\mathcal{B}_{1}, \mathcal{B}_{2}\right)$ and there exists $W \in \mathcal{L}\left(\mathcal{B}_{1}^{\prime}, \mathcal{B}_{2}^{\prime}\right)$ such that $W \in G_{T}(X)$, for each $X \in$ $\mathcal{L}\left(\mathcal{B}_{1}, \mathcal{B}_{2}\right)$.

Constant LFR can be characterized in terms of the corresponding operator.

Proposition 1 [9, Proposition 1]. For a matrix $T$ the following conditions are equivalent:

(i) $G_{T}$ is constant.

(ii) There exists an operator $W$ in $\mathcal{L}\left(\mathcal{B}_{1}^{\prime}, \mathcal{B}_{2}^{\prime}\right)$ such that

$$
T=\left(\begin{array}{cc}
T_{11} & T_{12} \\
W T_{11} & W T_{12}
\end{array}\right) .
$$

The main result of [8] characterizes, in the Hilbert space case, operators $T$ for which 0 is an interior point in the domain of $G_{T}$; (the case of other interior points can be reduced to this using 'change of coordinates'; see [8, Remark 3.8]). 
THEOREM 1 [8, Theorem 3.2]. If $\mathcal{B}_{1}, \mathcal{B}_{2}, \mathcal{B}_{1}^{\prime}, \mathcal{B}_{2}^{\prime}$ are Hilbert spaces, then an LFR $G_{T}$ is defined on some ball $B(r)$, where $r>0$, if and only if at least one of the following conditions is satisfied.

(a) $G_{T}$ is constant.

(b) The operator $T_{11}$ is bounded below.

(c) The operator $T_{11}$ is left Fredholm and $T(\mathcal{B}) \cap \mathcal{B}_{2}^{\prime}=\{0\}$.

We prove (Theorem 2(1)) that for separable Banach spaces the conditions of Theorem 1 are still necessary. They become sufficient either when $G_{T}$ is constant, or when the image of $T_{11}$ is complemented (Theorem 2(2)). On the other hand, there exist Banach spaces $\mathcal{B}=\mathcal{B}_{1} \oplus \mathcal{B}_{2}, \mathcal{B}^{\prime}=\mathcal{B}_{1}^{\prime} \oplus \mathcal{B}_{2}^{\prime}$ and operators $T_{11}, T_{12}, T_{21}$, such that (i) $T_{11}$ is bounded below, (ii) there exists $T_{22}$ such that the obtained LFR $G_{T}$ is non-constant and $\operatorname{dom} G_{T}=\mathcal{L}\left(\mathcal{B}_{1}, \mathcal{B}_{2}\right)$ (iii) changing $T_{22}$ we get an LFR whose domain does not contain any $B(r)$ with $r>0$ (Theorem 2(3)). This example shows that, in the Banach space case, the condition (b) is not sufficient, and that the complementability of the image of $T_{11}$ is not necessary, even in the non-constant case.

The second purpose of this paper is to continue the study (initiated in [9]) of the problem: to what extent do linear fractional relations share the property of entire functions described by the Liouville's theorem: "a bounded entire function is constant"? Bounded linear fractional relations are defined in the following way.

Definition 2. A linear fractional relation $G_{T}$ is called bounded if

$$
\sup _{X \in \operatorname{dom}\left(G_{T}\right)} \inf _{Y \in G_{T}(X)}\|Y\|<\infty .
$$

It was discovered in [9] that the existence of bounded everywhere defined nonconstant linear fractional relations depends on the geometry of the Banach spaces $\mathcal{B}_{1}, \mathcal{B}_{2}, \mathcal{B}_{1}^{\prime}, \mathcal{B}_{2}^{\prime}$. In this connection we introduce the following terminology.

Definition 3. A quadruple $\left(\mathcal{B}_{1}, \mathcal{B}_{2}, \mathcal{B}_{1}^{\prime}, \mathcal{B}_{2}^{\prime}\right)$ is called a Liouville quadruple if for each $T: \mathcal{B}_{1} \oplus \mathcal{B}_{2} \rightarrow \mathcal{B}_{1}^{\prime} \oplus \mathcal{B}_{2}^{\prime}$ such that $\operatorname{dom} G_{T}=\mathcal{L}\left(\mathcal{B}_{1}, \mathcal{B}_{2}\right)$ and $G_{T}$ is bounded, the relation $G_{T}$ is constant.

In Theorem 3 we find a class of non-Liouville quadruples, generalizing [9, Theorem 2].

All examples of Liouville quadruples found in [9] are such that the canonical image of $\mathcal{B}_{2}^{\prime}$ in its second dual is complemented. In Theorem 4 we show that if $\mathcal{B}_{1}, \mathcal{B}_{2}$, and $\mathcal{B}_{1}^{\prime}$ are Hilbert spaces, then $\left(\mathcal{B}_{1}, \mathcal{B}_{2}, \mathcal{B}_{1}^{\prime}, \mathcal{B}_{2}^{\prime}\right)$ is a Liouville quadruple for an arbitrary Banach space $\mathcal{B}_{2}^{\prime}$.

2. Interior points in the domain of an LFR between Banach spaces. Our generalization of Theorem 1 to the Banach space case is as follows.

THEOREM 2.

(1) If the spaces $\mathcal{B}_{1}$ and $\mathcal{B}_{2}$ are separable and $B(r) \subset \operatorname{dom} G_{T}$ for some $r>0$, then at least one of the following conditions is satisfied:

(a) $G_{T}$ is constant.

(b) $T_{11}$ is bounded below.

(c) $T_{11}$ is left Fredholm and $T(\mathcal{B}) \cap \mathcal{B}_{2}^{\prime}=\{0\}$. 
(2) If $\operatorname{im} T_{11} \subset \mathcal{B}_{1}^{\prime}$ is complemented, then each of the conditions (b) and (c) implies that $\operatorname{dom} G_{T}$ contains $B(r)$ for some $r>0$.

(3) Let $\mathcal{X}$ and $\mathcal{U}$ be Banach spaces such that there exists an isomorphic embedding $A: \mathcal{X} \rightarrow \mathcal{U}$ with an uncomplemented image. Let $\mathcal{B}_{1}=\mathcal{B}_{2}=\mathcal{X}, \mathcal{B}_{1}^{\prime}=\mathcal{U}, \mathcal{B}_{2}^{\prime}=$ $\mathcal{U} \oplus \mathcal{X}$. Let $T_{11}=A, T_{21}=A \oplus 0, T_{12}=0$. Then, for some choices of $T_{22} \neq 0$ the linear fractional relation $G_{T}$ is non-constant and $\operatorname{dom} G_{T}=\mathcal{L}\left(\mathcal{B}_{1}, \mathcal{B}_{2}\right)$, whereas 0 is not an interior point of $\operatorname{dom} G_{T}$ for some other choices of $T_{22}$.

Proof. Part (1). Suppose that $T$ is such that $B(r) \subset \operatorname{dom} G_{T}$ for some $r>0$ and $G_{T}$ is non-constant.

Proposition 2. Let $\mathcal{B}_{1}$ be a separable Banach space. If an operator $S: \mathcal{B}_{1} \rightarrow \mathcal{B}_{1}^{\prime}$ is not left Fredholm, then there exists a closed subspace $L$ of infinite codimension in $\mathcal{B}_{1}$, such that $S(L)$ is dense in $S\left(\mathcal{B}_{1}\right)$.

Proof. Since $\mathcal{B}_{1}$ is separable, then there exists a fundamental minimal system $\left\{x_{i}\right\}_{i=1}^{\infty}$ in the space $\operatorname{cl}\left(S\left(\mathcal{B}_{1}\right)\right)$; (see [14, p. 43]). The construction of [14, Proposition 1.f.3] shows that we may assume that $x_{i} \in S\left(\mathcal{B}_{1}\right)$. Let $y_{i} \in \mathcal{B}_{1}$ be such that $S y_{i}=x_{i}$. We may and shall assume that

$$
\sum_{i=1}^{\infty}\left\|y_{i}\right\|<\frac{1}{4}
$$

(If the condition (2) is not satisfied for the originally selected sequence $\left\{y_{i}\right\}_{i=1}^{\infty}$, we multiply $x_{i}$ and $y_{i}$ by suitable constants.) We need the following version of the wellknown (see e.g. [14, Proposition 1.a.9]) perturbation result.

Lemma 1. There exists a sequence $\left\{\delta_{i}\right\}_{i=1}^{\infty}, \delta_{i}>0$, such that, if $u_{i} \in S\left(\mathcal{B}_{1}\right)$ satisfy $\left\|u_{i}-x_{i}\right\|<\delta_{i}$, then $\left\{u_{i}\right\}_{i=1}^{\infty}$ is also fundamental in $\operatorname{cl}\left(S\left(\mathcal{B}_{1}\right)\right)$.

Proof. Let $\left\{x_{i}^{*}\right\}_{i=1}^{\infty} \subset\left(\operatorname{cl}\left(S\left(\mathcal{B}_{1}\right)\right)\right)^{*}$ be such that $\left(\left\{x_{i}\right\}_{i=1}^{\infty},\left\{x_{i}^{*}\right\}_{i=1}^{\infty}\right)$ is a biorthogonal system. Let $\delta_{i}>0$ be such that $\sum_{i=1}^{\infty} \delta_{i}\left\|x_{i}^{*}\right\|<1$, and let $u_{i}$ be such that $\left\|u_{i}-x_{i}\right\|<\delta_{i}$. Consider the operator $T: \operatorname{cl}\left(S\left(\mathcal{B}_{1}\right)\right) \rightarrow \operatorname{cl}\left(S\left(\mathcal{B}_{1}\right)\right)$ given by

$$
T x=x+\sum_{i=1}^{\infty} x_{i}^{*}(x)\left(u_{i}-x_{i}\right) .
$$

Observe that

(1) $T x_{i}=u_{i}$.

(2) $\left\|T-I_{\mathrm{cl}\left(S\left(\mathcal{B}_{1}\right)\right)}\right\|<1$.

The condition (2) implies that $T$ is invertible. Hence $\left\{u_{i}\right\}_{i=1}^{\infty}$ is the image of a fundamental system under an invertible operator, therefore $\left\{u_{i}\right\}_{i=1}^{\infty}$ is fundamental.

Recall that a sequence $\left\{z_{i}\right\}_{i=1}^{\infty}$ in a Banach space is called basic if for each vector $z$ in the closure of the linear span of $\left\{z_{i}\right\}_{i=1}^{\infty}$ there is a unique sequence of scalars $\left\{a_{i}\right\}_{i=1}^{\infty}$ so that $z=\sum_{i=1}^{\infty} a_{i} z_{i}$. We refer to [14, Chapter 1] for information on this notion.

LEMMA 2. There exists a normalized basic sequence $\left\{z_{i}\right\}_{i=1}^{\infty} \subset \mathcal{B}_{1}$ with basic constant 2, such that $\left\|S z_{2 i}\right\|<\delta_{i}$.

Proof. Since $S$ is not left Fredholm, then for each subspace $C$ of finite codimension in $\mathcal{B}_{1}$ and for each $\delta>0$, there exists $v \in C$ such that $\|v\|=1$ and $\|S v\|<\delta$. We get the 
result by combining this observation with the well-known approach to the construction of basic sequences [14, pp. 4-5].

We continue the proof of Proposition 2. We show that the subspace $L=$ $\operatorname{cl}\left(\operatorname{lin}\left(\left\{z_{2 i}+y_{i}\right\}_{i=1}^{\infty}\right)\right) \subset \mathcal{B}_{1}$ satisfies all the conditions of Proposition 2.

1. Infinite codimension. Indeed, the condition (2) combined with the well-known result on stability of basic sequences [14, Proposition 1.a.9(i)] implies that the sequence

$$
z_{1}, z_{2}+y_{1}, z_{3}, z_{4}+y_{2}, \ldots, z_{2 i-1}, z_{2 i}+y_{i}, \ldots
$$

is a basic sequence in $\mathcal{B}_{1}$. Hence, its even terms span a subspace of infinite codimension in $\mathcal{B}_{1}$.

2. Density of $S(L)$. Indeed, $S(L)$ contains the vectors $u_{i}=S\left(z_{2 i}+y_{i}\right)=S\left(z_{2 i}\right)+x_{i}$. The choice of vectors $z_{2 i}$ (see Lemma 2) implies that the $u_{i}$ satisfy the condition of Lemma 1. By Lemma 1 the system $\left\{u_{i}\right\}_{i=1}^{\infty}$ is fundamental in $\operatorname{cl}\left(S\left(\mathcal{B}_{1}\right)\right)$. Hence $S(L)$ is dense in $S\left(\mathcal{B}_{1}\right)$.

REMARK. A Hilbert space analogue of Proposition 2 was proved in [8] using results on quasi-complements from [5], [6], and [17]. Since the proof in [8] uses duality, the approach of [8] does not seem to be suitable for the non-reflexive case. This is why we use the double-perturbation approach of the paper [15] instead.

We continue our proof of Theorem 2. Assume that $T_{11}$ is not left Fredholm. By Proposition 2 there exists a subspace $L \subset \mathcal{B}_{1}$ of infinite codimension such that $T_{11}(L)$ is dense in $T_{11}\left(\mathcal{B}_{1}\right)$. We introduce $K \in \mathcal{L}\left(\mathcal{B}_{1}, \mathcal{B}_{2}\right)$ as an operator satisfying the following conditions:

(I) $\left.K\right|_{L}=0$,

(II) $K$ maps $\mathcal{B}_{1}$ onto a dense subspace of $\mathcal{B}_{2}$.

To construct such an operator we observe that the quotient space $\mathcal{B}_{1} / L$ is infinite dimensional, hence (see $\left[\mathbf{1 4}\right.$, p. 43]) there are sequences $\left\{x_{i}\right\}_{i=1}^{\infty} \subset\left(\mathcal{B}_{1} / L\right)$ and $\left\{x_{i}^{*}\right\}_{i=1}^{\infty} \subset$ $\left(\mathcal{B}_{1} / L\right)^{*}$ such that $x_{i}^{*}\left(x_{j}\right)=\delta_{i, j}$ (Kronecker delta). Let $\left\{b_{i}\right\}_{i=1}^{\infty}$ be a dense sequence in $\mathcal{B}_{2}$ (we use the separability of $\mathcal{B}_{2}$ ). We introduce an operator $D: \mathcal{B}_{1} / L \rightarrow \mathcal{B}_{2}$ by

$$
D(x)=\sum_{i=1}^{\infty} \frac{x_{i}^{*}(x)}{2^{i}\left\|x_{i}^{*}|||| b_{i}\right\|} b_{i} .
$$

Observe that $D\left(x_{i}\right)$ is a scalar multiple of $b_{i}$. Hence $\operatorname{im} D$ is dense in $\mathcal{B}_{2}$. Let $Q: \mathcal{B}_{1} \rightarrow \mathcal{B}_{1} / L$ be a quotient mapping. Then $K=D Q$ satisfies the conditions (I) and (II).

Fix $t \leq r /\|K\|$. We have assumed that $B(r) \subset \operatorname{dom} G_{T}$. Hence there exists $W_{t}$ satisfying

$$
T_{21}+T_{22} t K=W_{t}\left(T_{11}+T_{12} t K\right)
$$

and $W_{0}$ such that

$$
T_{21}=W_{0} T_{11}
$$

From (3) and (I) we get

$$
T_{21} x=W_{t} T_{11} x, \quad \forall x \in L .
$$


Since $T_{11}(L)$ is dense in $T_{11}\left(\mathcal{B}_{1}\right)$, we conclude that

$$
W_{t} x=W_{0} x, \quad \forall x \in T_{11}\left(\mathcal{B}_{1}\right) .
$$

Hence

$$
T_{21}=W_{t} T_{11}
$$

Subtracting (4) from (3) we get

$$
T_{22} t K=W_{t} T_{12} t K
$$

Since the image of $K$ is dense in $\mathcal{B}_{2}$, we get

$$
T_{22} x=W_{t} T_{12} x, \quad \forall x \in \mathcal{B}_{2} .
$$

We get a contradiction with the assumption that $G_{T}$ is non-constant. This contradiction implies that $T_{11}$ is left Fredholm.

To finish the proof of Part (1) it remains to show that if $T_{11}$ is not bounded below, then $T(\mathcal{B}) \cap \mathcal{B}_{2}^{\prime}=0$. The argument of this part of the proof is the same as in the Hilbert space case. (See the end of the proof of Theorem 3.2 in [8].) We reproduce it for the convenience of the reader.

Suppose that $T(x \oplus y) \in \mathcal{B}_{2}^{\prime}$. This means that $T_{11} x+T_{12} y=0$. We need to show that this implies that $T_{21} x+T_{22} y=0$.

The operator $T_{11}$ is left Fredholm and is not bounded below. Therefore $\operatorname{ker} T_{11} \neq\{0\}$. Hence we can choose $z \in \operatorname{ker} T_{11}$ in such a way that $r\|z+x\| \geq\|y\|$. Then there exists $K \in B(r)$ satisfying $K(z+x)=y$. Hence $z+x \in \operatorname{ker}\left(T_{11}+T_{12} K\right)$.

The LFR $G_{T}$ is defined on $B(r)$. Hence there exist $W$ satisfying

$$
T_{21}+T_{22} K=W\left(T_{11}+T_{12} K\right)
$$

and $W_{0}$ satisfying

$$
T_{21}=W_{0} T_{11}
$$

From (5) we get that $z+x \in \operatorname{ker}\left(T_{21}+T_{22} K\right)$. It follows from (6) that $z \in \operatorname{ker} T_{21}$. Hence

$$
0=\left(T_{21}+T_{22} K\right)(z+x)=T_{21} z+T_{21} x+T_{22} K(z+x)=T_{21} x+T_{22} y,
$$

and we are done.

PART (2). Case (b). Suppose that $T_{11}$ is bounded below and its image is complemented. We show that in such a case $\operatorname{dom} G_{T} \supset B(r)$ for some $r>0$.

Let $P: \mathcal{B}_{1}^{\prime} \rightarrow \operatorname{im} T_{11}$ be a projection. The operator

$$
W_{0}=T_{21}\left(\left.T_{11}\right|_{\text {im } T_{11}}\right)^{-1} P \in \mathcal{L}\left(\mathcal{B}_{1}^{\prime}, \mathcal{B}_{2}^{\prime}\right)
$$

satisfies $W_{0} T_{11}=T_{21}$ and hence $W_{0} \in G_{T}(0)$. It is now clear that it is enough to verify that $\operatorname{im}\left(T_{11}+T_{12} K\right)$ is complemented if $\|K\|$ is small enough.

To see this we observe that $\operatorname{im}\left(T_{11}+T_{12} K\right)$ is the image of $\operatorname{im}\left(T_{11}\right)$ under the operator $\left(T_{11}+T_{12} K\right)\left(\left.T_{11}\right|_{\operatorname{im} T_{11}}\right)^{-1} P$ and, hence, under the operator $I+T_{12} K\left(\left.T_{11}\right|_{\operatorname{im} T_{11}}\right)^{-1} P$. If the operator $K$ has sufficiently small norm, then $\left\|T_{12} K\left(T_{11} \lim _{11}\right)^{-1} P\right\|<1$, and, by the well-known observation, the operator $I+$ $T_{12} K\left(\left.T_{11}\right|_{\operatorname{im} T_{11}}\right)^{-1} P$ is an automorphism of $\mathcal{B}_{1}^{\prime}$. Hence it maps complemented subspaces onto complemented subspaces. 
Case (c). Suppose now that $T_{11}$ is a left Fredholm operator with a non-trivial finite dimensional kernel $F$. First we prove that $\operatorname{im}\left(T_{11}+T_{12} K\right)$ is complemented if $\|K\|$ is small enough.

Let $C$ be a complement of $F$ in $\mathcal{B}_{1}$. Then $\left.T_{11}\right|_{C}$ is bounded below. Repeating the argument used in the case (b) we get that $\left(T_{11}+T_{12} K\right)(C)$ is a complemented subspace of $\mathcal{B}_{1}^{\prime}$, if $\|K\|$ is small enough. Observe that

$$
\left(T_{11}+T_{12} K\right)\left(\mathcal{B}_{1}\right)=\operatorname{lin}\left(\left(T_{11}+T_{12} K\right)(C) \cup T_{12} K(F)\right) .
$$

Hence $\left(T_{11}+T_{12} K\right)\left(\mathcal{B}_{1}\right)$ is complemented as a sum of a complemented subspace and a finite-dimensional subspace.

It is also known (see [14, p. 78]) that $\left(T_{11}+T_{12} K\right)$ is left Fredholm when $\|K\|$ is small enough. It remains to show that the complementability of the image of $\left(T_{11}+T_{12} K\right)$ and the left-Fredholmness of $\left(T_{11}+T_{12} K\right)$ for $K \in B(r)$ together with the condition $T(\mathcal{B}) \cap \mathcal{B}_{2}^{\prime}=\{0\}$ imply that $B(r) \subset \operatorname{dom} G_{T}$. The condition $T(\mathcal{B}) \cap \mathcal{B}_{2}^{\prime}=\{0\}$ implies that $x \in \operatorname{ker}\left(T_{11}+T_{12} K\right)$ (or $\left.T(x \oplus K x) \in \mathcal{B}_{2}^{\prime}\right)$ implies $T(x \oplus K x)=0$. This means that $\left(T_{21}+T_{22} K\right) x=0$. We get $\operatorname{ker}\left(T_{21}+T_{22} K\right) \supset \operatorname{ker}\left(T_{11}+T_{12} K\right)$.

Let $F_{K}$ be the kernel of $T_{11}+T_{12} K$. Let $C_{K}$ be a complement of $F_{K}$ in $\mathcal{B}_{1}$. Then $\left.\left(T_{11}+T_{12} K\right)\right|_{C_{K}}$ is invertible. Denote by $P$ a continuous linear projection onto im $\left(T_{11}+\right.$ $\left.T_{12} K\right)$. Let

$$
W=\left(T_{21}+T_{22} K\right)\left(\left.\left(T_{11}+T_{12} K\right)\right|_{C_{K}}\right)^{-1} P
$$

The identity

$$
W\left(T_{11}+T_{12} K\right) x=\left(T_{21}+T_{22} K\right) x
$$

can be easily verified for $x \in C_{K}$. The condition $\operatorname{ker}\left(T_{21}+T_{22} K\right) \supset \operatorname{ker}\left(T_{11}+T_{12} K\right)$ implies that it is satisfied, also, for $x \in F_{K}$. Hence

$$
W\left(T_{11}+T_{12} K\right)=\left(T_{21}+T_{22} K\right)
$$

and $B(r) \subset \operatorname{dom} G_{T}$.

PART (3). Let $T_{22}: \mathcal{B}_{2} \rightarrow \mathcal{B}_{2}^{\prime}$ be any non-zero operator that can be factored through $\ell_{\infty}$ (any other injective space can be used for this example), that is, there exist $F$ : $\mathcal{B}_{2} \rightarrow \ell_{\infty}$ and $G: \ell_{\infty} \rightarrow \mathcal{B}_{2}^{\prime}$ such that $G F=T_{22}$. We claim that with this choice of $T_{22}$ the LFR $G_{T}$ is defined everywhere. Since $T_{21}=I_{\mathcal{B}_{1}^{\prime} \rightarrow \mathcal{B}_{2}^{\prime}} T_{11}$, where $I_{\mathcal{B}_{1}^{\prime} \rightarrow \mathcal{B}_{2}^{\prime}}$ is the natural embedding of $\mathcal{U}$ into $\mathcal{U} \oplus \mathcal{X}$, and $T_{12}=0$, it is enough to establish, that, for an arbitrary $X: \mathcal{B}_{1} \rightarrow \mathcal{B}_{2}$, the operator $T_{22} X$ is equal to $Z T_{11}$ for some $Z \in \mathcal{L}\left(\mathcal{B}_{1}^{\prime}, \mathcal{B}_{2}^{\prime}\right)$. Observe that the existence of such $Z$ is equivalent to the possibility of extension of the operator $T_{22} X A^{-1}$ from $A \mathcal{X}$ (its natural domain) to $\mathcal{U}$. This possibility follows from the requirement that $T_{22}=G F$. Indeed, it implies $T_{22} X A^{-1}=G F X A^{-1}$. By the injectivity of $\ell_{\infty}$ the operator $F X A^{-1}$ can be extended to an arbitrary Banach space containing $A \mathcal{X}$ as a subspace. Since $T_{12}=0$ and $T_{22} \neq 0$, then, by Proposition $1, G_{T}$ is non-constant.

Now let $T_{22}=0 \oplus I_{\mathcal{X}}$. To finish the proof of the theorem it suffices to prove that the operators $X=\varepsilon I_{\mathcal{X}}, \varepsilon \neq 0$, are not in $\operatorname{dom} G_{T}$. That is, we need to prove that there is no $Y \in \mathcal{L}\left(\mathcal{B}_{1}^{\prime}, \mathcal{B}_{2}^{\prime}\right)$ satisfying $Y T_{11}=T_{21}+T_{22} \varepsilon I_{\mathcal{X}}$ for $\varepsilon \neq 0$. Indeed, the subspace $\left(T_{21}+T_{22} \varepsilon I_{\mathcal{X}}\right)(\mathcal{X}) \subset \mathcal{B}_{2}^{\prime}$ is complemented, whereas the subspace $T_{11}(\mathcal{X}) \subset$ $\mathcal{B}_{1}^{\prime}=\mathcal{U}$ is uncomplemented, and a bounded linear operator cannot map isomorphically an uncomplemented subspace onto a complemented one. In more detail: since both 
$T_{11}$ and $T_{21}+T_{22} \varepsilon I_{\mathcal{X}}$ are isomorphic embeddings, then the restriction to $T_{11}(\mathcal{X})$ of a continuous linear operator $Y$ satisfying $Y T_{11}=T_{21}+T_{22} \varepsilon I_{\mathcal{X}}$ should be a bijection between $T_{11}(\mathcal{X})$ and $\left(T_{21}+T_{22} \varepsilon I_{\mathcal{X}}\right)(\mathcal{X})$. Also its inverse, let us denote it by $\left(\left.Y\right|_{T_{11}(\mathcal{X})}\right)^{-1}$, should also be continuous. Denote by $P: \mathcal{B}_{2}^{\prime} \rightarrow\left(T_{21}+T_{22} \varepsilon I_{\mathcal{X}}\right)(\mathcal{X})$ a continuous linear projection onto $\left(T_{21}+T_{22} \varepsilon I_{\mathcal{X}}\right)(\mathcal{X})$. It is easy to check that $\left(\left.Y\right|_{T_{11}(\mathcal{X})}\right)^{-1} P Y: \mathcal{U} \rightarrow \mathcal{U}$ is a continuous linear projection onto $T_{11}(\mathcal{X})$. The existence of such a projection contradicts our assumption.

3. More non-Liouville quadruples. Our next purpose is to describe a class of non-Liouville quadruples. The result obtained is a strengthening of [9, Theorem 2].

THEOREM 3. Let $\mathcal{X}$ be a Banach space with the bounded approximation property and such that its canonical image in $\mathcal{X}^{* *}$ is uncomplemented.

Let $\mathcal{U}$ be a Banach space satisfying the condition: there is an isomorphic embedding $A: \mathcal{X} \rightarrow \mathcal{U}$ such that

(a) there is a bounded net $B_{\alpha}: \mathcal{U} \rightarrow A(\mathcal{X})$ of finite rank operators such that the restrictions $\left\{\left.B_{\alpha}\right|_{A(\mathcal{X})}\right\}_{\alpha}$ converge to $I_{A(\mathcal{X})}$ in the strong topology,

(b) $A(\mathcal{X})$ is uncomplemented in $\mathcal{U}$.

Let $\mathcal{Y}$ be such that each linear continuous operator $X: \mathcal{X} \rightarrow \mathcal{Y}$ is compact, and there exists a linear continuous operator $D: \mathcal{Y} \rightarrow \mathcal{X}$ with dense range.

Let $\quad \mathcal{B}_{1}=\mathcal{X}, \quad \mathcal{B}_{2}=\mathcal{Y} \oplus \mathcal{A}_{2}, \quad \mathcal{B}_{1}^{\prime}=\mathcal{X} \oplus \mathcal{U} \oplus \mathcal{A}_{1}^{\prime}, \quad \mathcal{B}_{2}^{\prime}=\mathcal{X} \oplus \mathcal{X} \oplus \mathcal{A}_{2}^{\prime}, \quad$ where $\mathcal{A}_{2}, \mathcal{A}_{1}^{\prime}, \mathcal{A}_{2}^{\prime}$ are arbitrary Banach spaces.

Then $\left(\mathcal{B}_{1}, \mathcal{B}_{2}, \mathcal{B}_{1}^{\prime}, \mathcal{B}_{2}^{\prime}\right)$ is a non-Liouville quadruple.

REMARK 1. A space $\mathcal{U}$ satisfying the conditions (a) and (b) of Theorem 3 exists for each $\mathcal{X}$ satisfying the conditions above, one of such spaces is $\mathcal{X}^{* *}$, with $A$ being the canonical embedding of $\mathcal{X}$ into $\mathcal{X}^{* *}$ and $B_{\alpha}$ being the second conjugates of the operators strongly approximating the identity on $\mathcal{X}$.

REMARK 2. Many pairs $(\mathcal{X}, \mathcal{Y})$ of Banach spaces satisfying this condition are known, starting with the well-known result of H. R. Pitt [16] that shows $\mathcal{X}=c_{0}$ and $\mathcal{Y}=\ell_{p}(1 \leq p<\infty)$ satisfy the condition. See, also [14, p. 76].

Proof. Let

$$
\begin{aligned}
T_{11}(x) & =(x, 0,0), \\
T_{21}(x) & =(x, 0,0), \\
T_{12}(y, a) & =(D y, A D y, 0), \\
T_{22}(y, a) & =(D y, D y, 0) .
\end{aligned}
$$

Let $G_{T}$ be the corresponding LFR. It is enough to prove the following result.

LEMMA 3. $G_{T}$ is a non-constant, everywhere defined, bounded LFR.

Proof. To show that $G_{T}$ is non-constant, assume the contrary. By Proposition 1 this assumption implies that there exists $W: \mathcal{B}_{1}^{\prime} \rightarrow \mathcal{B}_{2}^{\prime}$ such that $T_{21}=W T_{11}$ and $T_{22}=W T_{12}$. From the definitions of $T_{i j}$ we immediately get:

$$
\begin{gathered}
W(x, 0,0)=(x, 0,0)(\forall x \in \mathcal{X}) . \\
W(D y, A D y, 0)=(D y, D y, 0)(\forall y \in \mathcal{Y}) .
\end{gathered}
$$


Since the image of $D$ is dense in $\mathcal{X}$, the condition (8) implies that

$$
W(x, A x, 0)=(x, x, 0)(\forall x \in \mathcal{X}) .
$$

Subtracting the equation (7), we get

$$
W(0, A x, 0)=(0, x, 0)(\forall x \in \mathcal{X}) .
$$

Let $P: \mathcal{B}_{2}^{\prime} \rightarrow \mathcal{X}$ be defined by $P(x, y, a)=y$, and let $I_{\mathcal{U}, \mathcal{B}_{1}^{\prime}}: \mathcal{U} \rightarrow \mathcal{B}_{1}^{\prime}$ be the natural embedding operator. Then the composition $A P W I_{\mathcal{U}, \mathcal{B}_{1}^{\prime}}$, acting along the sequence $\mathcal{U} \rightarrow \mathcal{B}_{1}^{\prime} \rightarrow \mathcal{B}_{2}^{\prime} \rightarrow \mathcal{X} \rightarrow \mathcal{U}$, is a projection onto $A(\mathcal{X})$. The existence of this projection contradicts the assumption that $A(\mathcal{X})$ is uncomplemented. Hence $G_{T}$ is non-constant.

To show that $G_{T}$ is bounded and $\operatorname{dom} G_{T}=\mathcal{L}\left(\mathcal{B}_{1}, \mathcal{B}_{2}\right)$, we consider an arbitrary $X \in \mathcal{L}\left(\mathcal{B}_{1}, \mathcal{B}_{2}\right)$. Let $X(x)=\left(X_{1}(x), X_{2}(x)\right)$, where $X_{1}: \mathcal{X} \rightarrow \mathcal{Y}$ and $X_{2}: \mathcal{X} \rightarrow \mathcal{A}_{2}$. Then

$$
\begin{gathered}
\left(T_{11}+T_{12} X\right)(x)=\left(x+D X_{1}(x), A D X_{1}(x), 0\right), \\
\left(T_{21}+T_{22} X\right)(x)=\left(x+D X_{1}(x), D X_{1}(x), 0\right) .
\end{gathered}
$$

We need to show that for each $X$ there is an operator $Y_{X}: \mathcal{B}_{1}^{\prime} \rightarrow \mathcal{B}_{2}^{\prime}$ satisfying

$$
Y_{X}\left(x+D X_{1}(x), A D X_{1}(x), 0\right)=\left(x+D X_{1}(x), D X_{1}(x), 0\right),
$$

and such that there exists an estimate of $\left\|Y_{X}\right\|$ from above that does not depend on $X$.

We are going to find $Y_{X}$ in the form,

$$
Y_{X}(x, u, a)=\left(x, Z_{X}(x, u), 0\right),
$$

and so, we need to find $Z_{X}$ with norm bounded by a constant depending only on $A$ and $\left\{B_{\alpha}\right\}$, and such that

$$
Z_{X}\left(x+D X_{1}(x), A D X_{1}(x)\right)=D X_{1}(x)
$$

By one of the conditions of Theorem 3, the operator $X_{1}$ is compact. Hence, for each $\varepsilon>0$, there exists $\alpha$ such that $\left\|\left(I_{\mathcal{U}}-B_{\alpha}\right) A D X_{1}\right\|<\varepsilon$. From here we conclude that there exists an absolute constant $C<\infty$, such that for each $X_{1}$ we can choose $\alpha$ in such a way, that there exists $E=E_{X_{1}, \alpha}: \mathcal{X} \rightarrow \mathcal{X}$ satisfying

$$
E\left(I_{\mathcal{X}}+\left(I_{\mathcal{X}}-A^{-1} B_{\alpha} A\right) D X_{1}\right)=\left(I_{\mathcal{X}}-A^{-1} B_{\alpha} A\right) D X_{1}
$$

and $\|E\| \leq C$.

We let

$$
Z_{X}(x, u)=E\left(x-A^{-1} B_{\alpha} u\right)+A^{-1} B_{\alpha} u .
$$

It is clear that $\left\|Z_{X}\right\|$ is bounded from above by a constant which depends only on $\left\|A^{-1}\right\|$ and $\sup _{\alpha}\left\|B_{\alpha}\right\|$. It remains to verify that (9) is satisfied. The verification is straightforward.

4. More Liouville quadruples. Positive Liouville-type results for LFR in [9] (see [9, Theorem 1]) use the condition “ $\mathcal{B}_{2}^{\prime}$ is complemented in its second dual space". Our next purpose is to prove a positive Liouville-type result of a somewhat different nature. 
THEOREM 4. Let $\mathcal{B}_{1}=\mathcal{B}_{1}^{\prime}=\mathcal{B}_{2}=\ell_{2}$ and $\mathcal{B}_{2}^{\prime}$ be an arbitrary Banach space. Then $\left(\mathcal{B}_{1}, \mathcal{B}_{2}, \mathcal{B}_{1}^{\prime}, \mathcal{B}_{2}^{\prime}\right)$ is a Liouville quadruple.

Proof. Let $T$ be such that $G_{T}$ is everywhere defined and bounded. We need to show that $G_{T}$ is constant. By Proposition 1, in order to do this, we need to find $W: \mathcal{B}_{1}^{\prime} \rightarrow \mathcal{B}_{2}^{\prime}$ satisfying $W T_{11}=T_{21}$ and $W T_{12}=T_{22}$. For each subspace $L$ of infinite codimension in $\mathcal{B}_{1}$ and each $M \in \mathbb{N}$ there exists an operator $X=X_{L, M}: \mathcal{B}_{1} \rightarrow \mathcal{B}_{2}$, such that $\left.X\right|_{L}=0$ and the unit balls satisfy $X\left(B_{\mathcal{B}_{1}}\right) \supset M B_{\mathcal{B}_{2}}$. Let $Y=Y_{L, M} \in G_{T}\left(X_{L, M}\right)$ with $\sup _{L, M}\left\|Y_{L, M}\right\|=C<\infty$. (Such $Y_{L, M}$ exist because we assume that $G_{T}$ is bounded.)

By Theorem 2, if $G_{T}$ is defined everywhere, then either $G_{T}$ is constant, or $T_{11}$ is left Fredholm. Therefore, to prove Theorem 4 it suffices to consider the case in which $T_{11}$ is left Fredholm. We introduce the subspaces $K_{1, L}=T_{11}(L) \subset \mathcal{B}_{1}^{\prime}$ and $K_{2}=$ $\operatorname{cl}\left(T_{12}\left(\mathcal{B}_{2}\right)\right) \subset \mathcal{B}_{1}^{\prime}$.

LeMmA 4. Let $K_{L}=\operatorname{cl}\left(K_{1, L}+K_{2}\right)$. The restrictions $\left\{\left.Y_{L, M}\right|_{K_{L}}\right\}_{M=1}^{\infty}$ converge in the strong operator topology as $M \rightarrow \infty$ and the limit $Y_{L}: K_{L} \rightarrow \mathcal{B}_{2}^{\prime}$ of this sequence satisfies

(1) $Y_{L} T_{11}(x)=T_{21}(x)(\forall x \in L)$,

(2) $Y_{L} T_{12}(x)=T_{22}(x)\left(\forall x \in \mathcal{B}_{2}\right)$.

Proof. Since

$$
Y_{L, M}\left(T_{11}+T_{12} X_{L, M}\right)=T_{21}+T_{22} X_{L, M}
$$

and $\left.X_{L, M}\right|_{L}=0$, we have that

$$
Y_{L, M} T_{11}(x)=T_{21}(x) \forall x \in L \forall M \in \mathbb{N} \text {. }
$$

Let $z \in \mathcal{B}_{2}$ be of norm 1 . Then there is $x \in B_{\mathcal{B}_{1}}$ such that $X_{L, M} x=M^{\prime} z$, for some $M^{\prime} \geq M$. From

$$
Y_{L, M}\left(T_{11}+T_{12} X_{L, M}\right) x=\left(T_{21}+T_{22} X_{L, M}\right) x
$$

we get

$$
\left\|Y_{L, M} T_{12} M^{\prime} z-T_{22} M^{\prime} z\right\| \leq C\left\|T_{11}\right\|+\left\|T_{21}\right\|
$$

Hence

$$
\lim _{M \rightarrow \infty}\left\|Y_{L, M} T_{12} z-T_{22} z\right\|=0
$$

Therefore $\left\{\left.Y_{L, M}\right|_{K_{L}}\right\}_{M=1}^{\infty}$ is a bounded sequence of operators that is convergent on a dense subset of $\operatorname{cl}\left(K_{1, L}+K_{2}\right)$. Hence it is convergent in the strong topology. It is clear that its strong limit satisfies (1) and (2).

We shall "paste" the desired operator $W$ from two "pieces". These are the operators $Y_{L_{1}}$ and $Y_{L_{2}}$, constructed according to Lemma 4 for suitably chosen subspaces $L_{1}, L_{2} \subset$ $\mathcal{B}_{1}$.

Let $K_{1}=T_{11}\left(\mathcal{B}_{1}\right)$. Let $P: K_{1} \rightarrow K_{2}^{\perp}$ be the restriction to $K_{1}$ of the orthogonal projection of $\mathcal{B}_{1}^{\prime}$ onto $K_{2}^{\perp}$. 
Lemma 5. There exists an orthogonal decomposition $K_{1}=R_{1} \oplus R_{2}$ of $K_{1}$ into a sum of two infinite dimensional subspaces such that the images $P\left(R_{1}\right)$ and $P\left(R_{2}\right)$ are also orthogonal.

Proof. Let $S: K_{2}^{\perp} \rightarrow K_{1}$ be any isometric embedding. Then, by the Polar Decomposition (see, e.g. [2, p. 15]), $S P=W|S P|$, where $W$ is a partial isometry with the initial space $(\operatorname{ker} P)^{\perp}$ and the final space cl(im $(S P)$ ) (we use the terminology from [2]). Since $|S P|$ is a positive operator, then, by the spectral theorem (see, e.g. [2, $\S 10]$ ), there exists an orthogonal decomposition $K_{1}=R_{1} \oplus R_{2}$ such that $R_{1}$ and $R_{2}$ are infinite dimensional, and the subspaces $|S P| R_{1}$ and $|S P| R_{2}$ are orthogonal. Indeed, if the spectrum $\sigma(|S P|)$ of $|S P|$ is infinite, then we consider an infinite relatively open subset $F \subset \sigma(|S P|)$ whose complement $\sigma(|S P|) \backslash F$ also contains an infinite relatively open subset. Let $E$ be the spectral measure of $|S P|$, and let $\chi_{F}$ be the indicator function of $F$. It is clear that setting $R_{1}$ to be the image of the projection $\int \chi_{F} d E$, we get the desired decomposition. If the spectrum $\sigma(|S P|)$ is finite, then it consists of eigenvalues, and at least one of the corresponding eigenspaces is infinite dimensional. We decompose an infinite dimensional eigenspace into an orthogonal sum of infinite dimensional subspaces, and add the remaining eigenspaces to one of them. It is clear that in both cases we get the desired decomposition.

Since $W$ maps $\operatorname{cl}(\operatorname{im}|S P|)$ onto $\operatorname{cl}(\operatorname{im} S P)$ isometrically, then the orthogonality of the subspaces $|S P| R_{1}$ and $|S P| R_{2}$ implies the orthogonality of $S P\left(R_{1}\right)$ and $S P\left(R_{2}\right)$. Since $S$ is an isometric embedding of the space containing $P\left(K_{1}\right)$ then this, in turn, implies the orthogonality of $P\left(R_{1}\right)$ and $P\left(R_{2}\right)$.

Recall that our purpose is to find an operator $W \in \mathcal{L}\left(\mathcal{B}_{1}^{\prime}, \mathcal{B}_{2}^{\prime}\right)$, such that $W T_{11}=$ $T_{21}$ and $W T_{12}=T_{22}$.

Observe that since $P\left(R_{1}\right)$ and $P\left(R_{2}\right)$ are orthogonal, then $(I-P) R_{1}$ and $(I-P) R_{2}$ are also orthogonal. Hence $K_{2}$ and $K_{2}^{\perp}$ can be decomposed into the orthogonal sums $K_{2}=S_{1} \oplus S_{2}$ and $K_{2}^{\perp}=T_{1} \oplus T_{2}$ in such a way that $R_{1} \subset S_{1} \oplus T_{1}$ and $R_{2} \subset S_{2} \oplus T_{2}$. We define $W$ in two "pieces" as follows.

Let $L_{1}=T_{11}^{-1}\left(R_{1}\right)$ and $L_{2}=T_{11}^{-1}\left(R_{2}\right)$. The subspaces $L_{1}$ and $L_{2}$ are infinite dimensional and

$$
\mathcal{B}_{1}=\operatorname{lin}\left(L_{1} \cup L_{2}\right)
$$

In the case in which $T_{11}$ is left Fredholm, but is not bounded below, the subspaces $L_{1}$ and $L_{2}$ have finite dimensional intersection.

Let $Y_{L_{1}}: \operatorname{cl}\left(R_{1}+K_{2}\right) \rightarrow \mathcal{B}_{2}^{\prime}$ and $Y_{L_{2}}: \operatorname{cl}\left(R_{2}+K_{2}\right) \rightarrow \mathcal{B}_{2}^{\prime}$ be the operators whose existence is shown in Lemma 4. We consider the restrictions

$$
W_{L_{1}}=\left.Y_{L_{1}}\right|_{\mathrm{cl}\left(R_{1}+K_{2}\right) \cap S_{1} \oplus T_{1}}
$$

and

$$
W_{L_{2}}=\left.Y_{L_{2}}\right|_{\mathrm{cl}\left(R_{2}+K_{2}\right) \cap S_{2} \oplus T_{2}} .
$$

Since the spaces $S_{1} \oplus T_{1}$ and $S_{2} \oplus T_{2}$ are orthogonal, and the space $\mathcal{B}_{1}^{\prime}$ is a Hilbert space, then there exists an operator $W \in \mathcal{L}\left(\mathcal{B}_{1}^{\prime}, \mathcal{B}_{2}^{\prime}\right)$ that is a common extension of $W_{L_{1}}$ and $W_{L_{2}}$. It remains to show that $W$ is the desired operator. By construction we have

(i) $W T_{11}(x)=T_{12}(x) \forall x \in L_{1}$ and $\forall x \in L_{2}$. By (12), this implies $W T_{11}(x)=$ $T_{12}(x) \forall x \in \mathcal{B}_{1}$, and also we have hence $W T_{11}=T_{21}$. 
(ii) $W T_{12}(x)=T_{22}(x) \forall x \in T_{12}^{-1}\left(S_{1}\right)$ and $\forall x \in T_{12}^{-1}\left(S_{2}\right)$. Since $K_{2}=S_{1} \oplus S_{2}$, this implies that $W T_{12}(x)=T_{22}(x), \forall x \in \mathcal{B}_{2}$. Hence $W T_{12}=T_{22}$.

REMARK. The classes of Liouville quadruples and non-Liouville quadruples that were found in [9] and in the present paper are still far from being complementary classes. In particular, the following problem is open.

Problem. Let $\mathcal{B}_{2}^{\prime}$ be a Banach space whose canonical image in $\left(\mathcal{B}_{2}^{\prime}\right)^{* *}$ is uncomplemented. Does it follow that there exist $\mathcal{B}_{1}, \mathcal{B}_{2}$, and $\mathcal{B}_{1}^{\prime}$, such that $\left(\mathcal{B}_{1}, \mathcal{B}_{2}, \mathcal{B}_{1}^{\prime}, \mathcal{B}_{2}^{\prime}\right)$ is a non-Liouville quadruple?

\section{REFERENCES}

1. T. Ya. Azizov and I. S. Iokhvidov, Linear operators in spaces with indefinite metric and their applications (in Russian), Itogi Nauki Tekh., Ser. Mat. Anal. 17 (1979), 113-206; English translation: J. Soviet Math. 15 (1981), 438-490.

2. J. B. Conway, A course in operator theory, Graduate Studies in Mathematics, Vol. 21 (American Mathematical Society, Providence, R.I., 2000).

3. I. S. Iokhvidov, Banach spaces with a $J$-metric. $J$-nonnegative operators (in Russian) Dokl. Akad. Nauk SSSR 169 (1966) 259-261; English translation: Soviet Math. Dokl. 7 (1966), 896-899.

4. I. S. Iokhvidov, $J$-nondilating operators in a Banach space (in Russian), Dokl. Akad. Nauk SSSR 169 (1966), 519-522; English translation: Soviet Math. Dokl. 7 (1966), 962-965.

5. R. C. James, Quasicomplements, J. Approximation Theory 6 (1972), 147-160.

6. W. B. Johnson, On quasi-complements, Pacific J. Math. 48 (1973), 113-118.

7. V. A. Khatskevich, Fixed points of generalized linear-fractional transformations (in Russian), Izv. Akad. Nauk SSSR, Ser. Mat. 39 (1975), 1130-1141; English translation: Math. USSR-Izvestiya 9 (1975), 1069-1080.

8. V. A. Khatskevich, M. I. Ostrovskii, and V. S. Shulman, Linear fractional relations for Hilbert space operators, Math. Nachrichten 279 (2006), 875-890.

9. V. A. Khatskevich, M. I. Ostrovskii, and V. S. Shulman, An analogue of the Liouville theorem for linear fractional relations in Banach spaces, Bull. Australian Math. Soc. 73 (2006), 89-105.

10. V. A. Khatskevich, V. A. Senderov, and V. S. Shulman, On operator matrices generating linear fractional maps of operator balls, in Complex analysis and dynamical systems, Contemp. Math. 364 (2004), 93-102.

11. V. A. Khatskevich and V. S. Shulman, Operator fractional-linear transformations: convexity and compactness of image; applications, Studia Math. 116 (1995), 189-195.

12. M. G. Krein, A new application of the fixed-point principle in the theory of operators in a space with an indefinite metric, (in Russian), Dokl. Akad. Nauk SSSR 154 (1964), 1023-1026; English translation: Soviet Math. Doklady 5 (1964), 224-228.

13. M. G. Krein and Ju. L. Šmuljan, On linear-fractional transformations with operator coefficients (in Russian), Matematicheskie Issledovaniya (Kishinev) 2 (1967), no. 3, 64-96; English translation: Amer. Math. Soc. Transl., Ser. 2103 (1974), 125-152.

14. J. Lindenstrauss and L. Tzafriri, Classical Banach spaces, I-Sequence spaces. (SpringerVerlag, 1977).

15. M. I. Ostrovskii and V. P. Fonf, Operators with dense range and extensions of minimal systems (in Russian), Izvestiya vuzov. Matem. (1990), 45-47; English translation: Soviet Math. (Iz. VUZ) 34 (1990), 53-56.

16. H. R. Pitt, A note on bilinear forms, J. London Math. Soc. 11 (1936), 174-180.

17. A. N. Plichko, Selection of subspaces with special properties in a Banach space and some properties of quasicomplements (in Russian), Funktsional. Anal. i Prilozhen 15 (1981), 82-83; English translation: Funct. Anal. Appl. 15 (1981), 67-68. 